\title{
Editorial
}

\section{Vaginal Hysterectomy for non-prolapsed Uterus}

Hysterectomy is the most common operation performed by gynaecologists, next to caesarean section. In the continuous changing era of medical science no one can stick to older methods of surgical treatment. Scientists are always searching the easier, safer, more comfortable and more economic methods for both patients and surgeons. Traditionally various routes for removal of uterus have been used. Abdominal hysterectomy is undoubtedly the most popular with a $70: 30$ ratio for abdominal versus vaginal route ${ }^{1,2}$. In older days, the vaginal hysterectomy was limited only for the prolapsed uterus where uterus descends or comes outside and vaginal canal is lax enough to perform the surgery without any difficulties. For non descended uterus abdominal route was the route of choice. But with advancement of medical science and increased skill of surgeons, gynaecologists are moving from more invasive surgeries to less invasive one. So laparoscopic surgery replaced most of the abdominal cases. But total laparoscopic hysterectomy (TLH) requires highly skilled surgeon, special endoscopic equipments, trained medical and paramedical staff, skilled anaesthetist, long duration of operation and high cost. So vaginal hysterectomy has gained popularity to gynaecologists for benign uterine diseases where there is no need to inflict any injury in the abdominal wall.

Route of hysterectomy primarily depends on physician's choice. Factors to be considered in choosing the route for hysterectomy should include safety, cost-effectiveness, and the medical needs of the patients ${ }^{3}$. For non-descended vaginal hysterectomy following factors should be considered

- Surgeon's training and experience

- Accessibility of the uterus

- Extent of extrauterine disease

- Size and shape of the uterus

- Need for concurrent procedures like salpingooophorectomy

- Patient's preference.

In-spite of the fulfilling all of the above mentioned factors, it is not always feasible to do vaginal hysterectomy in all cases of non-descended uterus. There are some relative contraindications to vaginal hysterectomy, such as

- Enlarged uterus due to multiple and big fibroids and adenomyoma

- Nulliparity

- Narrow vagina

- Narrow pubic arch $\left(<90^{\circ}\right)$

- Fixed and immobile uterus due to adhesions

- Severe endometriosis

In practice choice of route depends upon surgeon's expertise and confidence and condition of the patient. Many nulliparous women and many women who have undergone caesarean section have sufficient vaginal capacity to allow a vaginal hysterectomy. As long as the surgeon can obtain adequate access for division of uterosacral and cardinal ligaments, the uterus can be mobilized sufficiently to allow vaginal extraction. Even when the uterus is enlarged due to fibroids, vaginal hysterectomy can be accomplished safely by means of morcellation, uterine bisection, wedge debulking or intramyometrial coring ${ }^{4}$.

\section{Advantages of vaginal hysterectomy:}

A cochrane review found that the vaginal route of hysterectomy has many advantages over abdominal and laparoscopic hysterectomy. It has shorter duration of hospital stay, faster return to normal activity, fewer febrile episodes or unspecified infections in comparison to abdominal hysterectomy. When it is compared with laparoscopic hysterectomy it has shorter operating time and it is non-injurious to the abdomen of patient as it performs through natural passage.

The VALUE study concluded that major haemorrhage, haematoma, ureteric injury, bladder injury, and anesthetic complications were more in laparoscopic assisted hysterectomy (LAVH) group when compared with abdominal and vaginal hysterectomies. In addition LAVH needed twice the time required for vaginal hysterectomy ${ }^{5}$. The study has supported the fact that there is significant reduction in complication rate in 
vaginal route than abdominal and recommended vaginal route as the primary route.

Though the skill and experience of the surgeon plays a pivotal role in determining the route of surgery, vaginal route should be the preferred one because it uses a natural route, it is smoother, no abdominal scar and safer operative procedure with satisfactory result than abdominal route either open or laparoscopic.

\section{Dr. Mosammat Rashida Begum}

Editor

\section{References:}

1. Sheth SS. Vaginal hysterectomy.In: PuriR, Malhotra N. eds. OperativeObstetrics and Gynaecology. New Delhi. Jaypee brothers medicalpublishers.2009. 499-510

2. Sheth SS, Paghdiwalla K P. In: Saraiya UB, Rao A K, Chateerjee A.eds. Principles and
Practice of obstetrics and Gynaecology.2nd ed.New Delhi. Jaypee brothers medical publishers.2003.374-30

3. Landeen LB, Bell MC, Hubert HB, Bennis LY, Kntsen-Laeson SS, Seshadri-Kreaden U. Clinical and cost comparisons for hysterectomy via abdominal, standard laparoscopic, vaginal and robot-assisted approaches. SD Med. 2011;64(6):197-9.

4. ACOG Committee Opinion No. 444: Choosing the route of hysterectomy for benign disease. Obstet Gynaecol. 2009; 114(5):1156-1158.

5. Mc Craker G, Hunter D, Morgan D, Price JH. Comparison of laparoscopic assisted vaginal hysterectomy, total abdominal hysterectomy and vaginal hysterectomy. Ulster Med J. 2006; 75(1):54-58. 